\title{
Linx
}

Revue des linguistes de l'université Paris X Nanterre

$51 \mid 2004$

Théories de l'écriture et pratiques scolaires

\section{Avec les nouvelles technologies, un rapport nouveau à l'écriture?}

Nicole Marty

\section{(2) OpenEdition}

1 Journals

Édition électronique

URL : http://journals.openedition.org/linx/210

DOI : $10.4000 / \operatorname{lin} \times .210$

ISSN : 2118-9692

Éditeur

Presses universitaires de Paris Nanterre

\section{Édition imprimée}

Date de publication : 1 décembre 2004

Pagination : 147-162

ISSN : 0246-8743

\section{Référence électronique}

Nicole Marty, « Avec les nouvelles technologies, un rapport nouveau à l'écriture? », Linx [En ligne], 51 | 2004, mis en ligne le 28 janvier 2011, consulté le 10 décembre 2020. URL : http://

journals.openedition.org/linx/210; DOI : https://doi.org/10.4000/linx.210 


\title{
Avec les nouvelles technologies, un rapport nouveau à l'écriture?
}

\author{
Nicole Marty \\ Académie de Paris \\ MoDyCo UMR 7114 CNRS - Université Paris $X$
}

\section{Introduction}

Les recherches dans le domaine de l'écriture des enfants ont longtemps porté sur l'analyse de textes écrits, dans leur état final. Les théories répertoriées à partir des années 80 (Hayes et Flower') ont ensuite mis l'accent sur les processus d'écriture pour essayer de dégager les invariants de la production écrite. C'est surtout sur les comportements de scripteurs, sur les opérations mentales que les linguistes, psycholinguistes et psychologues cognitivistes se sont penchés.

Pour donner du sens à l'écriture scolaire, en adoptant un éclairage culturel, dans le cadre de la didactique de la langue maternelle, il a aussi été nécessaire de faire appel, dans le champ des sciences humaines, à des sciences connexes, comme la philosophie, l'anthropologie, la sociolinguistique ou l'ethnographie.

Dans l'optique de donner du sens à nos travaux d'analyse linguistique de corpus d'enfants, oraux ou écrits, nous avons choisi d'écouter dix enseignants d'école élémentaire, parlant de leur pratique de l'écriture dans des environnements informatiques. Leur école se situe dans un quartier plutôt aisé de l'Ouest parisien où les élèves reçoivent, le plus souvent, une initiation à l'informatique à la maison, ce qui permet aux enseignants de se préoccuper davantage d'apprentissage de l'écrit avec l'ordinateur que de découverte des techniques.

Les enregistrements d'entretiens semi-directifs ont été transcrits et analysés dans le but de prendre en compte la parole de ces pionniers de l'écriture numérique et leurs représentations, afin de compléter les informations linguistiques issues de corpus

\footnotetext{
${ }^{1}$ Hayes J. R., Flower, L.S. (1980), Identifying the organisation of writing processes, in Cognitive processes in writing (L. W. Gregg et E. R. Steinberg, éds.), Hillsdale, Lawrence Erlbaum Ass., pp. 3-30.

Hayes J. R. (1995), Un nouveau modèle du processus d'écriture, in La production de textes. Vers un modèle d'enseignement de l'écriture (J. Y. Boyer et P. Raymond, dir.), Les Éditions Logiques, Montréal, pp. 49-72.
} 
écrits ou oraux d'enfants de cours moyen. L'étude de ces corpus (Marty 1991), a mis en évidence, essentiellement, des indications d'ordre métalinguistique sur l'écrit en cours de genèse, en salle informatique.

L'une des ambitions annexes est bien sûr, dans la sphère professionnelle qui est la nôtre, de faire évoluer ces pratiques enseignantes sur le terrain.

Les pratiques scolaires interrogent les théories de l'écriture, en créant des turbulences, car leur extrême complexité, impossible à appréhender dans un seul champ disciplinaire ${ }^{2}$, oblige au contraire les modèles à se juxtaposer et à s'allier. D'où la tentation de choisir, comme véhicules transitoires, des modèles issus de certaines disciplines, apportant les éclairages nécessaires à la perception de cette écriture scolaire.

\section{Choix de modèles}

Pour réaliser ces entretiens et les analyser dans le but de décrire et de comprendre les pratiques innovantes d'écriture, le modèle de l'ethnographie, adapté à l'écriture scolaire, a été privilégié.

Le travail du linguiste a porté, lui, sur l'enregistrement puis la transcription des discours sur l'écrit. Le travail d'enquête de l'ethnographe de l'écriture scolaire se centre davantage sur les contenus, à savoir les pratiques d'écriture, les usages et les représentations de ces usages. Ces représentations, à l'œuvre dans les réponses, sont ensuite à confronter aux réalités observées directement dans les classes. Tous les matériaux collectés viennent alimenter le travail du didacticien de la langue écrite.

Quelques mots sur l'ethnographie, discipline émergeante, plus développée dans le monde anglo-saxon qu'en France. Sophie Lambert ${ }^{3}$, dans un texte publié sur Internet, décrit l'ethnographie scolaire et en particulier la méthode de l'observation

\footnotetext{
2 Jacques Anis, dans son article « Le traitement de texte : écriture ou méta-écriture ? » in Ecriture et traitement de texte, Repères n ${ }^{\circ} 11,1995$, écrit à ce sujet: «On sait que les pratiques d'écriture constituent un objet trop complexe pour être appréhendé par une seule discipline scientifique. La psychologie cognitive, avec les travaux de Hayes et Flower, a proposé des modèles généraux, mais comme l'écrivent Sharples et Pemberton, « la limitation de cette approche est qu'elle n'aborde pas directement l'effet du médium sur l'écriture et n'explore pas l'espace des stratégies d'écriture possibles » (1988). D’autre part, les situations expérimentales, visant à dégager un très petit nombre de paramètres fins, appauvrissent souvent les processus d'écriture. La description des pratiques d'écriture réelles et des changements induits par l'apparition des technologies informatiques nécessite de compléter l'apport du laboratoire par le recours à des méthodes moins dures, telles que le questionnaire dans une population ciblée (réponses fermées et ouvertes), le témoignage et l'observation directe (y compris l'auto-observation)».

Jacques Anis se réfère donc, en ce qui le concerne, à la linguistique génétique pour l'étude de la «matérialité des processus d'écriture », à la graphématique, pour celle des graphèmes et des espaces graphiques, ainsi qu'à la sémiolinguistique.

3 «Un exemple, l'ethnographie de l'école», S. Lambert (chercheuse en Sciences de l'éducation et membre de l'équipe de l'ARFE-CURSUS). Article consulté sur Internet le 10 mai 2003.
} 
dite «participante» qui implique chercheurs et personnes observées. Les outils privilégiés sont les «conversations de terrain», même informelles, les « entretiens non structurés », les «questionnaires », «l'étude de documents », officiels ou personnels. Cette collecte de données est soumise à l'analyse et ouvre sur la théorisation qui permet classification, catégorisation, formulation de concepts. L'étape finale est l'écriture qui rend compte de ces recherches descriptives.

Dans cet ensemble de techniques d'enquête, a été retenue celle de l'« entretien non structuré », qui, d'après Sophie Lambert est le moyen le mieux adapté, dans des milieux complexes tels que l'école :
«Le présupposé fondamental de l'entretien non structuré est que sa dynamique interne, son déroulement libre, va faire surgir une vérité. Ce déroulement va déterminer aussi les questions de l'enquêteur : il devra se laisser porter par le fil de la conversation. Alors que dans la situation générale d'observation participante, la situation à explorer est déjà structurée, dans l'entretien ethnographique, il y a mise en place d'un dispositif particulier de rencontre, qui est le dispositif propre à l'entretien, et c'est à l'intérieur de ce dispositif construit qu'on va tenter de laisser jouer la spontanéité de l'enquêté ».

Dans le cas qui nous concerne, les questions posées aux enseignants n'étaient pas préparées à l'avance par le chercheur. L'entretien est ainsi resté ouvert aux propos des enquêtés, colorés par leur parcours individuel. Il a également été guidé par l'expérience du chercheur, acquise dans l'observation antérieure des usages scolaires. Il en résulte des entretiens non directifs, fortement interactifs, qui renseignent autant sur les représentations des enseignants que sur les présupposés théoriques de l'enquêteur. Ces corpus oraux transcrits, soumis à l'analyse qualitative ont permis de catégoriser les réponses, d'extraire des matériaux linguistiques, des données comportementales et posturales. Sans doute, aussi, quelques éléments du réel.

Les principales questions tournent autour d'une préoccupation essentielle : le rapport à l'écriture ${ }^{4}$ dans les classes de cours moyen a-t-il évolué avec l'introduction des ordinateurs? Si tel est le cas, quelles sont les tendances significatives repérées ? Quelles évolutions ces enseignants perçoivent-ils ? Comment envisagent-ils l'avenir ?

Dans l'ensemble, ces maittres d'école primaire, désignés par les directeurs comme étant les plus engagés dans la production d'écrit par ordinateur, apparaissent d'abord comme des enseignants plus experts que leurs collègues dans le domaine de l'écriture (avec ou sans ordinateur).

Cependant, chemin faisant, les technologies numériques, souvent restreintes à l'ordinateur, au traitement de texte et à Internet, ont modifié le rapport que ces maitres et ces élèves avaient avec la langue écrite. Les adultes ont bien saisi la valeur

\footnotetext{
${ }^{4}$ Sur ce thème du « rapport à » l'écriture, ici celui que les adultes et les enfants entretiennent avec l'écrit, cf. BARRÉ-DE MINIAC C. (2000), Le rapport à l'écriture. Aspects théoriques et didactiques, Presse universitaires du Septentrion.
} 
ajoutée que les techniques apportaient notamment à la révision des textes et à leur mise en forme.

Leurs récits insistent sur l'émergence de certains types d'écrits, sans doute induits par l'utilisation de certains logiciels (tels que les logiciels de PAO pour la production de journaux scolaires), dans des environnements particuliers : ainsi l'écrit, comme tout apprentissage scolaire, est bien dépendant du contexte didactique et des contraintes matérielles.

Ces enseignants s'attardent aussi sur les pratiques d'écriture de certains de leurs élèves, à la maison, et sur le regard que les parents portent sur ces usages. Plus que jamais, au moment où l'écrit circule plus librement entre l'école et la famille, comme, d'une manière générale, entre le monde du travail et la sphère domestique, par le canal de ces technologies, le milieu scolaire est conduit à s'interroger sur ses missions spécifiques. Apprendre aux élèves à écrire, paraît être la première d'entre elles. Cet apprentissage peut-il tirer parti de la fascination pour les écrans?

\section{Les difficultés rencontrées}

\section{Des observables en perpétuel mouvement}

Décrire les usages linguistiques dans le milieu scolaire ne va pas sans un certain inconfort. En effet, travailler sur des données linguistiques, orales et écrites, dans des environnements didactiques en perpétuel changement n’est pas une sinécure : rien à voir avec l'analyse de textes bien délimités, que ces corpus soient oraux ou écrits, garants d'une permanence de l'objet à étudier. La recherche en laboratoire est apparemment mieux balisée, prenant appui sur des modèles plus solidement constitués, ouvrant sur des théorisations plus affirmées.

La réalité observée, dans ce contexte, est changeante, fuyante, en évolution constante. Ainsi, tout évolue très vite: les élèves, le même élève, les modes pédagogiques, les enseignants, dans leur effort d'adaptation aux élèves, dans leurs références conceptuelles, au fil de leurs expériences, de leurs lectures, de leur formation, etc. Aucun moment de classe ne peut vraiment, de ce fait, être comparé à un autre. Pour compliquer l'affaire, le chercheur lui-même, impliqué dans une dynamique d'observation au long cours, évolue.

C'est pourquoi, dans le domaine qui nous intéresse, comme, sans doute, dans les sciences humaines en général, les procédures comparatistes, les données statistiques et, a fortiori, les généralisations, sont, semble-t-il, vouées en grande partie à l'échec.

L'observation patiente d'élèves en train d'écrire dans des environnements informatiques, depuis une dizaine d'années, donne cependant l'impression de repérer des usages, des tendances, des lignes de force. 


\section{Le «nez sur le guidon» ou le risque d'envol}

Les différentes phases de ce travail, à la recherche de données exploitables au plan linguistique, donnent parfois l'impression d'isoler, de cerner de près les objets linguistiques (observés lors des analyses de corpus oraux transcrits, notamment); parfois l'impression de faire un pas de côté, voire plusieurs, avec peut-être le risque de larguer les amarres, dans l'exploration d'autres univers théoriques : celui des sciences de l'éducation, de la sociologie, de la philosophie (observation des usages, des représentations, des « discours sur », etc.).

D'où l'impression d'une tension entre une tâche de linguiste, «le nez sur le guidon», parfois oublieux des contextes qui donnent sens aux observables, et une conduite plus papillonnante, musardant dans des univers interprétatifs plus larges, faisant courir le risque de se déconnecter du linguistique.

C'est cependant ce va-et-vient conceptuel qui me parait avoir donné une certaine cohérence à une culture linguistique et didactique, alliant expérience de l'enseignement et travaux de linguistique.

\section{Un foisonnement de modèles}

Le linguiste-didacticien n'est pas en peine pour trouver des modèles dans les différentes théories ${ }^{5}$ qu'il est amené à côtoyer. C'est plutôt à un excès de modèles qu'il est confronté, dans lesquels il va devoir choisir, établir des hiérarchies, trier.

- Certains modèles linguistiques d'abord: sémiolinguistique de l'écrit (Anis ${ }^{6}$, 2000) qui donne des outils pour analyser les évolutions affectant les langages écrits, du fait de l'apparition et du développement des technologies électroniques; sociolinguistique ${ }^{7}$, pour l'analyse des usages langagiers, dans l'institution scolaire.

- Des modèles utilisés en information-communication, utiles pour comprendre comment l'introduction de machines modifie les contenus scolaires, les comportements langagiers, l'écriture elle-même, le type d'accompagnement des apprenants par les formateurs...

- Des modèles des sciences de l'éducation (Linard, 2000; Reuter, 1996; Perriault, 2002 ; Barré-De Miniac, 1996, 2000) et des modèles de la didactique de la langue maternelle (Bronckart, Schneuwly, 1991).

\footnotetext{
${ }^{5}$ Principales théories de référence, en rapport avec la didactique, l'écriture, les technologies numériques : sciences de l'éducation (Linard, 2000) didactique de l'écriture (Reuter, 1998; Barré de Miniac, 2002 ; Plane 2001) ; génétique textuelle (Lebrave ; Fabre, 1997) ; sémiolinguistique de l'écrit (Anis, 2000)

${ }^{6}$ Anis J. (2000), «Vers une sémiolinguistique de l'écrit », in Linguistique de l'écrit, linguistique du texte, Revue LINX, n 43, Université Paris X - Nanterre, pp 29-42.

${ }^{7}$ Gadet F. (1997), Le français ordinaire, Armand Colin, Paris.
} 
- Des modèles sociologiques, ethnologiques. Dans ce cadre, le modèle d'ethnographie de l'école, développé par Sophie Lambert, en sciences de l'éducation, a plus particulièrement retenu notre attention. Le modèle de l'ethnographie scolaire utilisé ici, permet de faire un «arrêt sur image ». Les usages évoqués dans les discours peuvent cependant être en décalage avec les pratiques réelles.

Au bout du compte, les deux fils rouges, patiemment tressés dans l'action, articulant toutes ces données théoriques, sur le long terme, sont la linguistique et la didactique.

Découper quelques îlots, les décrire sous différents angles, jeter quelques ponts entre ces îlots ; éviter le quantitatif, nécessairement faux; préférer le qualitatif qui apporte quelques éclairages pour guider l'action des maîtres en retour. Dans ce contexte, les modèles se dégagent a posteriori, plus qu'ils ne guident la recherche en amont. Les concepts surgissent du terrain, puis sont contrôlés à la lumière de données ultérieures, on les renforce et on les reformule autrement.

\section{Les résultats de l'enquête}

\section{Les témoins : des enseignants experts}

Dix professeurs des écoles de Cycle 3, enseignant en CM1 ou en CM2, ont été sollicités pour témoigner sur leur pratique de classe. Les maitres retenus pour cette étude ont été désignés par les directeurs d'école, comme étant ceux qui utilisaient le plus les ordinateurs de l'école, ceux qui faisaient le plus écrire les élèves.

\section{Les entretiens réalisés ${ }^{8}$}

\begin{tabular}{|c|l|l|c|}
\hline Prénom & Sexe & Classe & $\begin{array}{c}\text { Code } \\
\text { témoin }\end{array}$ \\
\hline M.-C. G. & F & CM1-CM2 & T 1 \\
\hline D. M. & H & CM2 & T 2 \\
\hline C. R. & F & CM2 & T 3 \\
\hline I. G. & F & CM2 & T 4 \\
\hline D. E. & H & CM2 & T 5 \\
\hline S. F. & F & CM1 & T 6 \\
\hline P. C. & H & CM2 & T 7 \\
\hline C. N. & H & Directeur & T 8 \\
\hline J.-P. D. & H & CM1-CM2 & T 9 \\
\hline M. V. & F & CM1 & T 10 \\
\hline
\end{tabular}

NB : dans l'article, les enseignants seront désignés par leur code (ex : T 1).

\footnotetext{
${ }^{8}$ Je tiens à remercier les enseignants (désignés ici par des initiales) qui ont accepté de répondre à des questions sur leurs pratiques de classe.
} 
Dans l'ensemble, ce sont des maittres confirmés, ayant déjà un certain âge et une certaine expérience de l'enseignement. Ils ont, de plus, la réputation d'être «moteurs » dans ce domaine de l'informatique et de pratiquer des méthodes actives. Quelques-uns ont été formés sur les ordinateurs du plan IPT (informatique pour tous, 1985). Certains, mais pas tous, ont réfléchi à une didactique de l'écriture, inspirée des récentes théories linguistiques, telles que la génétique textuelle, par exemple.

Bien souvent, ils se sont formés sur le tas, en même temps que les élèves ou, mieux, chez eux, en achetant un ordinateur personnel. Ils ont parfois suivi des stages d'informatique.

\section{L'objectif premier: pratique de l'écriture ou utilisation de la machine?}

On s'aperçoit, en les écoutant parler, que ces enseignants, les plus avancés dans les pratiques d'écriture avec les TICE, sont tout simplement ceux qui ont tenté de mettre en place, tout d'abord, une pratique de l'écriture. L'informatique est venue après, en adjuvant, comme un outil de plus au service de l'écriture.

L'ordinateur est parfois utilisé comme une machine à écrire : l'apprentissage du clavier, de la dactylographie, prennent alors le pas sur la créativité linguistique, sur le rédactionnel, sur la communication écrite.

\section{Enseignant bomme ou femme: des différences dans l'utilisation de l'ordinateur?}

Parmi les témoins interviewés, $50 \%$ d'hommes (alors qu'il y a $80 \%$ de femmes dans l'enseignement du premier degré : les hommes sont donc sur-représentés dans cet échantillon). Ceux-ci utilisent souvent l'ordinateur pour faire écrire des journaux scolaires. Chez les femmes, les pratiques sont plus diversifiées, plus ponctuelles. Elles prennent parfois l'allure d'exercices, par exemple pour apprendre les lettres sur le clavier ou pour faire de la grammaire.

Mais ce qui frappe surtout, c'est que la technique et la passion sont plutôt du côté des hommes. Les femmes, elles, font davantage découvrir à leurs élèves l'informatique, parce que «c'est au programme», parce que c'est «moderne», dans la société d'aujourd'hui, parce que cet apprentissage sera utile pour plus tard :

T 6 J'étais équipée à la maison, depuis six ou sept ans. Pour moi, l'informatique n'est pas une grande passion. Je pense que l'on ne peut pas s'en passer, c'est tout. Cela rend des tas de services. Donc, quand j'ai eu l'ordinateur à la maison, mon mari s'en servait, les enfants aussi, on ne peut pas aller contre le progrès. Pour l'école, je savais que cela me rendrait des tas de services. Je l'utilise notamment pour mes préparations de classe, au quotidien...Même si je ne suis pas passionnée, cela me semblait important d'en faire profiter mes élèves. 


\section{Influence du passé de l'enseignant sur l'utilisation de l'ordinateur en classe}

On s'aperçoit que la formation de l'enseignant, dans le passé, a un poids non négligeable sur l'utilisation de l'ordinateur dans les pratiques d'écriture. Notamment quand la formation (ou le métier préalable, pas nécessairement dans l'Education nationale) a obligé la personne à utiliser l'ordinateur, dans les tâches bureautiques, et d'apprendre la dactylographie.

T 8 C'est grâce au service militaire que j'ai appris à écrire ainsi : j'ai été secrétaire du colonel ; il m'a bien expliqué comment il fallait présenter les courriers. Il fallait faire l'en-tête comme cela, indiquer l'objet, les pièces jointes, les références... Cela m'a donné un cadre. A l'époque on écrivait cependant à la main ou avec des machines électriques. C'était contraignant : on utilisait beaucoup les rubans correctifs.

D'autres ne sauront jamais taper...Citons le cas d'un enseignant qui ne possède pas lui-même cette compétence, alors qu'il favorise cet apprentissage en classe. Chez lui, il compte sur sa femme pour taper à sa place!

T 5 Moi, cela me bloque, j'ai du mal à taper vite. D'ailleurs, à la maison, c'est ma femme qui tape. Je lui dicte ou alors je lui explique ce que je veux et c'est elle qui tape. Elle me tape tous les projets, parce qu'elle tape beaucoup plus vite que moi. Donc, c'est un gain de temps énorme. On perd beaucoup de temps en tapant. Je ferai peut-être un stage pour apprendre à taper plus rapidement.

\section{Le degré d'utilisation de l'informatique par l'enseignant lui-même}

Tous les enseignants n'utilisent pas l'informatique de la même manière, à titre professionnel ou à titre personnel. Dans l'ensemble, tous se sont équipés chez eux pour préparer leurs cours et utilisent cet équipement de manière plus ou moins intensive. Ceux qui sont passionnés par l'informatique, notamment les hommes, ont beaucoup appris sur leur ordinateur personnel comme sur ceux de l'école.

On s'aperçoit, à la lecture des témoignages, que les enseignants se sont souvent débrouillés par leurs propres moyens. Il en résulte donc une grande variété de profils due aux histoires individuelles, aux contraintes matérielles rencontrées, aux formations pédagogiques antérieures. Cette variété se retrouve au niveau des types d'écrits pratiqués dans les classes concernées. Cependant quelques tendances générales se dessinent.

\section{Écriture, réécriture}

Les réflexions portent ici sur le dispositif mis en place par l'enseignant pour rendre possible l'écriture avec des ordinateurs, sur les rapports qu'entretiennent entre eux adultes et enfants dans ces situations, sur la référence à des théories, 
principalement linguistiques, que l'on devine en filigrane dans ce qui est dit de la pratique?

L'enseignante la plus en pointe est formatrice en IUFM. Elle connait donc les théories sous-jacentes qui ont vu naitre des pratiques d'écriture innovantes dans l'enseignement du premier degré :

T 1 Les élèves n'écrivent pas directement. Ils font un premier jet, on en tire des critères, un deuxième jet et après, une fois que le deuxième jet a été écrit et noté, ils vont sur l'ordinateur de fond de classe saisir leur texte. Celui-ci est à nouveau corrigé. Les textes sont assez bien saisis.

Je pense que l'outil informatique a le gros avantage de pouvoir retravailler le texte, de pouvoir supprimer des passages, pouvoir en rajouter... Quand on est sur la feuille de papier, c'est plus difficile. Pouvoir aérer son texte, faire des paragraphes, la belle présentation, le travail fini. C'est plus facile au niveau de la belle présentation que sur papier. C'est valorisant.

On retrouve une approche semblable, chez ce deuxième enseignant qui n'hésite pas à corriger plusieurs fois les écrits de ses élèves :

Le traitement texte est utilisé pour finaliser les productions écrites. Cela confirme les nombreux constats effectués dans les recherches antérieures :

T 3 J'utilise ces logiciels de traitement de texte. Je ne les utilise pas en création pour l'instant. Je les utilise pour finaliser un produit. Les élèves n'ont pas une production directe sur ordinateur. Pour l'instant, chacun écrit un texte ; un comité de lecture choisit ; après, on part en salle informatique et on met les textes en forme. Auparavant, ils ont été corrigés. On continue à corriger sur l'ordinateur car il y a quand même des modifications qui s'effectuent.

Ce processus pédagogique, avec un avant et un après, semble le plus fréquent chez les maitres qui ont longuement réfléchi à la didactique de l'écriture. L'écrit " papier », avec une correction préalable par le maitre, avant d'aller vers les machines, apparait comme la solution la plus cohérente pour gagner du temps, quand la salle informatique est située loin de la classe. La situation pourrait évoluer avec l'attribution d'un portable à chaque élève, comme cela se pratique déjà, à titre expérimental dans certaines régions ${ }^{10}$.

\section{Écrire pour être évalué}

Les élèves qui veulent écrire davantage, par exemple pour faire des comptes rendus ou des exposés, sont incités à le faire chez eux, sur l'ordinateur familial mais le

\footnotetext{
${ }^{9}$ Théories sous-jacentes : cf. note $\mathrm{n}^{\circ} 5$.

10 Dans les Landes, par exemple, des collégiens ont été équipés d'ordinateurs portables. Ils peuvent les emmener chez eux pour travailler. Par ce biais, les familles sont également concernées par l'initiation à l'outil informatique.
} 
maitre soupçonne souvent les parents de travailler à la place des enfants. L'écriture numérique ne permet pas de vérifier quel est l'auteur des écrits :

T 6 Je demande aux parents de ne pas taper pour eux. Je pense tout de même que papa et maman corrigent, parce que dans le cas d'un exposé, ils veulent que ce soit correct.

On devine à quel point cet enseignement de l'écriture, mené à bien par les parents, est en réalité élitiste, puisque, en suivant l'exemple parental, ces enfants, déjà équipés, peuvent se former à l'écriture « longue », directement sur ordinateur...

Certains enseignants pensent que l'écriture solitaire, devant l'ordinateur en classe, «c'est mieux pour vérifier l'apprentissage du traitement de texte et de l'orthographe ».

\section{Les types d'écrits, en relation avec les nouvelles technologies}

Dans les articles de certains didacticiens de l'écriture, la volonté du maître d'apprendre à l'élève à produire tel ou tel genre de texte est première. Les stratégies didactiques sont là pour mieux apprendre à produire tel type de texte ou de discours. L'hypothèse formulée ici, a contrario, est que l'outil (ordinateur et logiciel) induit par sa présence certains types de textes. Quels sont ceux qui ont été le plus souvent cités et qui sont privilégiés dans les usages observés? L'outil informatique, tant par ses caractéristiques techniques que par son mode d'utilisation en primaire, induit surtout des textes brefs, réunis parfois en recueil.

\section{Le journal scolaire}

Parmi les types d'écrits pratiqués dans les classes des enseignants concernés, le journal scolaire (journal de classe ou journal d'école), est de très loin l'écrit le plus en vogue. Sans doute parce que ce type d'écrit permet d'obtenir une production d'une certaine longueur en juxtaposant les travaux de plusieurs élèves.

C'est la production "phare» dans les témoignages recueillis. Le maitre fait écrire ses élèves pour faire connaître son travail aux collègues, aux parents, à l'inspection... Quand le journal devient celui de l'école entière c'est bien souvent sous l'impulsion du directeur ou d'un maitre "pionnier».

\section{La correspondance scolaire par Internet, grâce à la messagerie}

Elle est citée par une seule enseignante ( $\mathrm{T}$ 1), sans doute parce que l'utilisation des messageries n'est pas encore passée dans les mœurs scolaires: les textes doivent être corrigés avant d'être envoyés.

Le mail est mal vu car il est souvent émaillé de fautes d'orthographe et difficilement contrôlable avant envoi. C'est donc une mauvaise vitrine de l'enseignement, pour des maitres soucieux de l'image de leur pédagogie :

T 2 On communique ainsi de manière occasionnelle, c'est vrai que ce n'est pas un outil que l'on utilise dans le cadre scolaire : autant le texte saisi sur ordinateur peut être valorisé, autant si l'on écrit de manière un peu hâtive un texte truffé de fautes, 
quelque part ce n'est pas acceptable. Ce n'est pas fait pour aller dans le sens des apprentissages que l'on essaie de promouvoir : avoir une bonne syntaxe, écrire des phrases correctes, avoir une bonne orthographe...

Cette communication ne se pratique en fait que lorsque les enfants sont en classe de découverte avec le maître car les parents sont friands de nouvelles.

\section{L'écriture de poèmes}

Compte tenu du temps imparti en salle informatique (une heure par semaine, au mieux), les textes créés sont souvent très courts. La poésie est alors un genre privilégié, à la fois pour la rapidité d'écriture et pour la calligraphie, le traitement de texte permettant les fantaisies graphiques.

\section{Des fiches de lecture}

Autre type de texte bref, la fiche de lecture, exercice éminemment scolaire, fait pour motiver les autres à lire (mais décourageant parfois le lecteur-scripteur lui-même, qui le perçoit comme un pensum associé à la lecture)

\section{Retravailler le texte, le corriger}

\section{Le correcteur orthographique}

Le traitement de texte semble avoir pour principale fonction de recueillir du texte mais aussi pour principal avantage de pouvoir le traiter. Le correcteur orthographique, l'une des fonctionnalités appréciée des usagers adultes, est en réalité fort peu utilisé dans les classes.

La raison tient principalement à la présence du logiciel de traitement de texte Works qui ne possède pas cette fonction, dans les écoles de la Ville de Paris. Cette situation est critiquée par l'ensemble des utilisateurs qui auraient préféré Word.

Dans l'un des témoignages, le maittre envisage de faire réfléchir les élèves sur la nature du soulignement :

T 5 Le correcteur montre à l'enfant qu'il y a un souci qui peut être de différentes natures : majuscule oubliée, fautes d'orthographe, un nom propre non reconnu par la machine, un espace oublié, etc.

Je n'ai pas encore approfondi cette dimension avec les élèves mais je sens que c'est une dimension très importante. Travailler avec les enfants sur ce sujet: est-ce une véritable faute d'orthographe? On essaie de comprendre l'erreur, de la corriger. Un certain nombre d'enfants sont déjà capables de sélectionner le mot mal orthographié, de cliquer sur le correcteur orthographique, pour voir ce que l'ordinateur propose à la place... 

réécriture

Le traitement de texte est motivant pour la révision du texte, pour la

Cette plus grande facilité des opérations de révision, encourageante pour les élèves, est évoquée par presque tous les enseignants :

T 10 Sur le cahier, sur une feuille, ils rechignent davantage. Avec l'ordinateur, c'est davantage un jeu.

T 2 Le but essentiel c'est de valoriser le travail des élèves pour qu'ils se rendent compte que le texte prend une autre dimension quand il est bien présenté, de manière plus normative, ressemblant à ce qu'ils peuvent lire dans le journal, dans un recueil de textes, etc.

\section{Les corrections textuelles sont très rares}

Les corrections effectuées sont des corrections de surface, plutôt ponctuelles. Cela s'explique en partie par le peu de temps dont disposent les élèves et par la brièveté de leur texte qui ne nécessite pas une gestion textuelle très élaborée.

\section{Le poids déterminant des contraintes matérielles et organisationnelles dans la production écrite}

L'utilisation de la salle informatique, dans l'enquête, a été opposée à celle des ordinateurs en fond de classe. Les modes d'accès à tous ces équipements sont des plus divers. Les maîtres concernés par l'enquête s'en servent souvent plus que les autres. Certains ont même installé leur classe à côté de la salle informatique et ajoutent, de surcroît, des machines de récupération dans leur classe.

\section{La salle informatique, c'est dépassé dans ce quartier}

T 2 Quand on travaille en réseau, c'est beaucoup plus difficile de créer un dossier, de le rentrer etc., on complexifie. Alors que, en fond de classe, c'est plus simple. En fait, la salle informatique était un outil pour initier les enfants à la pratique de l'ordinateur. Maintenant, on est au-delà... peut-être, dans ce quartier.

T 6 Dans la salle informatique, c'est compliqué, parce que ceux qui sont au milieu sont tentés de regarder ceux qui sont devant les ordinateurs et la qualité du travail n'est pas bonne. Autant rester en classe pour essayer de les faire travailler de manière différenciée. Mais là, en salle informatique, leur attention est plus dispersée. classe

Les maîtres les plus débrouillards utilisent salle d'informatique et fond de

Ils s'arrangent pour obtenir la classe à côté de la salle informatique. Cette dernière, une fois la porte ouverte, devient donc une annexe :

T 9 Comme la salle informatique est juste à côté de la mienne, je peux travailler pendant ce temps, dans ma salle de classe... Les élèves sont en production tout à fait autonome. 


\section{Le travail par demi groupe en salle informatique}

L'équipement d'une salle informatique a obligé les maitres à délaisser par moments la pédagogie frontale et collective pour faire travailler les élèves en deux groupes (ou plus), dans une approche plus différenciée de l'enseignement. On les sent parfois gênés d'avoir à gérer tous les groupes en même temps.

\section{Le fond de classe}

Le fond de classe permet la liberté, la souplesse d'utilisation et une pédagogie plus différenciée. Les maitres qui ont goûté à ce dispositif, avec des ordinateurs performants et des logiciels de qualité, disent bien qu'ils ne peuvent plus s'en passer.

Cela les oblige cependant à être plus créatifs dans l'organisation spatiale et temporelle, à fonctionner davantage par ateliers. La configuration optimale du fond de classe est semble-t-il de deux ou trois ordinateurs, dont l'un est connecté à Internet. Les élèves vont y taper à tour de rôle, seuls ou par petits groupes, ou bien ils vont y consulter la documentation numérique.

T 1 J'avais quatre ordinateurs en fond de classe quand j'étais à Strasbourg et je trouve que le dispositif de fond de classe donne plus de liberté. On doit aller en salle informatique de telle heure à telle heure, et ce n'est pas forcément le moment où l'enfant a envie de saisir son texte.

T 5 Nous avons un ordinateur en fond de classe, très performant, cette année. C'est un cadeau. Un parent d'élève nous l'a amené...

Moi qui étais contre l'informatique en fond de classe, j'arrive à être convaincu que l'on peut faire du bon travail. Je pense que si l'on avait trois appareils de ce type, cela permettrait à un petit groupe de travailler pendant que les autres font autre chose.

\section{Les logiciels utilisés}

Le recensement des logiciels utilisés par les maitres pour les travaux d'écriture de leurs élèves permet de constater une réduction des choix par rapport à ce qui avait été initialement prévu, lors de la création des salles informatiques. L'informatique, exogène à ses débuts, s'est progressivement intégrée aux activités. L'utilisation actuelle, plus conviviale, est nettement plus «littéraire », davantage tournée vers les tâches d'écriture et de lecture.

Les choix des enseignants se portent massivement vers Word, Internet, et vers les encyclopédies numériques. Après décantation, seuls certains logiciels, détournés de leur usage bureautique, le plus souvent « ouverts », restent en usage.

\section{Faut-il apprendre la dactylographie aux élèves?}

La question "Faudrait-il entraîner les élèves à la dactylographie?» n'est pas neutre. Elle renvoie les enseignants à leurs propres compétences et pratiques dans le domaine.

Ceux qui ne sont pas experts le regrettent. Ces maitres, dans l'ensemble, se sont formés par eux-mêmes et introduisent parfois en classe des logiciels d'apprentissage 
de la dactylographie. Le risque existe de voir les élèves taper beaucoup plus vite qu'eux, en peu de temps... D'ailleurs, certains enfants apprennent par eux-mêmes, chez eux et tapent leurs textes très vite, ce qui constitue un plus pour leur vie future.

T 10 Ils apprennent très vite. Pour eux, c'est un jeu. On apprend principalement à taper avec certains doigts, ils apprennent à placer leurs mains sur la barre du milieu et ils gagnent du temps. La mise en page se fait très vite, je suis très étonnée. Ils sont capables de jouer avec les polices d'écriture, de mettre des espaces... Cela ne leur pose aucun problème.

Quand on pose la question à ces maitres de cycle 3, cependant, ils estiment que les programmes scolaires ne laissent pas de temps pour cet enseignement. Certes, il faudrait dispenser celui-ci aux enfants, mais bien en amont, dans la scolarité, par exemple en maternelle.

La priorité reste l'écriture manuscrite. Le plaisir d'écrire à la main est plusieurs fois évoqué, non sans une certaine nostalgie.

\section{L'ordinateur à la maison}

\section{Le nombre d'élèves équipés chez eux}

Le nombre d'élèves qui ont un ordinateur à la maison, dans ce quartier, paraît relativement élevé. Parmi eux, il faudrait distinguer ceux qui ont un ordinateur personnel et ceux qui utilisent celui des parents. Dans ce dernier cas, l'usage est moins fréquent. Les rares enfants non équipés souffrent de cette situation. Le fossé «numérique » se creuse sans doute du fait de ces différences d'équipement.

\section{L'ordinateur à la maison, concurrent ou extension de la classe?}

On retrouve chez les élèves le phénomène constaté chez les adultes, à savoir la porosité entre sphère de travail et sphère domestique. Les élèves les plus zélés n’hésitent pas à emporter du travail à la maison pour taper leur fiche de lecture, saisir un texte, préparer un exposé.

Ceux qui sont ainsi équipés et connectés chez eux tapent généralement plus vite que les autres. Ils dépassent techniquement le maitre. Ils deviennent même des «tuteurs » pour ceux qui ont encore besoin d'une initiation à la saisie des textes au cycle 3 .

Les maitres ont un domaine de compétence qu'ils revendiquent: ils sont les seuls à apprendre aux élèves à écrire, au sens rédactionnel du terme, avec ou sans ordinateur. Ils ne veulent pas que l'école perde ses missions essentielles, dans une société de l'information où l'apprentissage s'effectue aussi hors de ses murs. A la maison, d'ailleurs, pensent les maitres, les enfants jouent :

T $7 \mathrm{Il}$ y a un décalage entre que l'on peut en faire à l'école et ce que, eux, en font à la maison. Eux, l'utilisent pour jouer, principalement. Les parents ont l'impression que leurs enfants se servent de l'ordinateur, alors qu'ils jouent. 


\section{Et Internet?}

Les écoles élémentaires ont été équipées d'une connexion à Internet, lors de l'installation des salles informatiques ou des fonds de classe. En cas de choix d'une salle informatique, seule celle-ci bénéficie d'une connexion. Mais quand c'est la classe qui est connectée, la navigation sur Internet, la consultation de documents ou d'encyclopédies en ligne, grâce aux moteurs de recherche, l'utilisation de messageries, s'effectuent plus souplement, sans inutiles pérégrinations.

Les enseignants ont cependant des inquiétudes au sujet de l'intrusion de la publicité ou de la pornographie par le canal d'Internet. On peut témoigner de la difficulté à maîtriser ces aspects quand une douzaine de postes sont allumés. Des filtres ont été installés, pas toujours efficaces. Ils rendent souvent la connexion difficile, voire impossible.

Les chemins doivent donc être sérieusement balisés, les sites explorés par les enseignants au préalable, les questions, pour les requêtes, préparées avec soin. La solution la plus éducative consiste sans doute à parler de la nature des documents trouvés sur Internet et à développer l'esprit critique.

Concernant l'avenir de ces technologies, les réponses sont prudentes. Les maitres ne veulent pas abandonner des objectifs essentiels : apprendre aux élèves à écrire à la main, aider les plus en difficulté à produire des textes... Il considèrent parfois le passé - ce temps où l'on n'écrivait qu'à la main - avec un peu de nostalgie.

\section{Conclusion}

Cette enquête, de type qualitatif, sur le mode des études ethnographiques, a permis d'avancer dans la réflexion: elle a confirmé certaines intuitions émises précédemment, lors de recherches à partir de corpus écrits ou oraux d'écoliers. Des tendances se sont dessinées qui demanderaient bien sûr à être confirmées dans des travaux à plus grande échelle.

L'enquête a eu aussi le mérite de mettre en évidence les représentations des maitres sur l'écriture avec ordinateur, dans un milieu donné et d'en donner une photographie à un moment donné.

L'étude de ces représentations n'a été possible qu'avec l'éclairage de modèles linguistiques et didactiques, eux-mêmes puisés à différentes sources, rencontrées au fil des lectures. Elle donne des éléments pour un travail «d'observation participante » ${ }^{11}$, de collecte de données et de description des usages de l'écrit dans le milieu considéré. Tel est l'objet de travaux inspirés par une linguistique centrée sur l'écriture scolaire et les environnements didactiques qui lui donnent sens.

\footnotetext{
${ }^{11}$ L'« observation participante » est l'une des méthodes utilisées par l'ethnologue Malinowski (18841942).
} 


\section{BIBLIOGRAPHIE DANS LES DOMAINES DE LA LINGUISTIQUE ET DE LA DIDACTIQUE DE LA LANGUE ÉCRITE :}

Anis, J., Temporal-Marty, N. (éds) (1990) Ecriture, Informatique, Pédagogies, Collection Microsavoirs, CNDP, Paris.

MARTY, N. (1991) «Quand les enfants parlent entre eux de traitement de texte et d'écriture », Actes du colloque interdisciplinaire Texte et ordinateur, les mutations du lire-écrire, Université de Paris X - Nanterre, Editions de l'espace européen.

MARTY, N. (janvier-mars 1991) L'écrit dans l'oral, Etudes de linguistique appliquée, nº 81, Didier Erudition, Paris.

MARTY, N. (juin 2000) «Des enfants, des ordinateurs, des maittres. L'école maternelle s'empare des nouvelles technologies ", Education enfantine, $\mathrm{n}^{\circ} 10$, Nathan, Paris.

MARTY, N. (novembre 2000) "Technologies numériques et pratiques d'écriture en classe: quelles évolutions », in Dossier: Langue et multimédia (coordonné par N. Marty), Journal des Instituteurs, $\mathrm{n}^{\circ} 3$, Nathan.

ANis, J., MARTy, N. (éds) (2000) Lecture-écriture et nouvelles technologies, Collection de l'ingénierie éducative, CNDP, Paris.

MARTY, N. (décembre 2000) «Ecouter les élèves quand ils parlent d'ordinateur », in Les TICE et l'école, Dossiers de l'ingénierie éducative, $\mathrm{n}^{\circ} 33$, CNDP.

MARTY, N. (13 novembre 2001) Les textos, un danger pour l'orthographe?, Education.com, Le magazine, rubrique Multimédia : http ://www.enseignants.com/mag/article

MARTY N. (mars 2003) «La maitrise du langage est-elle facilitée par la fréquentation des écrans ? ", Dossiers de l'ingénierie éducative, $\mathrm{n}^{\circ}$ 42, CNDP, Paris.

MARTy N. (décembre 2003) Pratiques d'écriture à l'école primaire: l'apport des nowvelles technologies, Thèse de doctorat sous la direction du Professeur Jacques Anis, Sciences du langage, Paris X - Nanterre. 